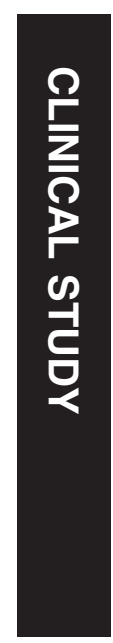

\title{
Analysis of Th17- associated cytokines in tears of patients with dry eye syndrome
}

${ }^{1}$ Department of Ophthalmology, Wuxi No. 2, People's Hospital, Nanjing Medical University, Wuxi, China

${ }^{2}$ Department of Cataract, Zhongshan Ophthalmic Centre, Guangzhou, China

${ }^{3}$ Key Laboratory of Nuclear Medicine, Ministry of Health, Jiangsu Key Laboratory of Molecular Nuclear Medicine, Jiangsu Institute of Nuclear Medicine, Wuxi, China

${ }^{4}$ Save Sight Institute, University of Sydney, Sydney, NSW, Australia

Correspondence:

$X$ Tan or S Sun

Department of

Ophthalmology, Wuxi No. 2, People's Hospital, ZhongShan Road, Wuxi 214000, China.

Tel: +86138 14247452;

Fax: +86 51085508775

E-mail: doctxh001@

gmail.com or

sxuefeng@vip.sina.com

Received: 8 May 2013 Accepted in revised form: 21 January 2014 Published online: 7 March 2014

\begin{abstract}
Purpose To determine the levels of Th17associated cytokines, particularly interleukin (IL)-17 and IL-22 in tears of patients with dry eye syndrome.

Methods Tear samples were collected from

20 healthy volunteers, 20 dry eye (DE) patients with non-Sjögren's syndrome (NSSDE) and $20 \mathrm{DE}$ patients with Sjögren's syndrome (SSDE). Symptom questionnaire was self-administered and multiple dry eye disease (DED)-related clinical tests were performed. The levels of IL-17 and IL-22 in tears were measured by enzyme-linked immunosorbent assay.

Results The levels of IL-17 and IL-22 were significantly increased in tears of DE patients compared with those of controls and also higher in SSDE patients compared with those of NSSDE patients $(P<0.05)$. Moreover, the levels of IL-17 and IL-22 were positively correlated with questionnaire score and keratopathy score but negatively correlated with tear film break-up time and Schirmer I test in both NSSDE and SSDE patients $(P<0.05)$.

Conclusions The levels of IL-17 and IL-22 in tears were significantly increased in DE patients, which were associated with the disease severity. Therefore, Th17 cellassociated cytokines, particularly IL-17 and IL-22, may have important roles in the immunopathogenesis of the DED.
\end{abstract}

Eye (2014) 28, 608-613; doi:10.1038/eye.2014.38; published online 7 March 2014

\section{Introduction}

Dry eye disease (DED) is a complex and multifactorial disease with decreased tear secretion or increased tear evaporation. ${ }^{1}$

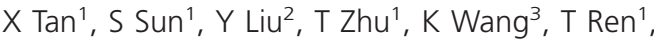
$\mathrm{Z} \mathrm{Wu}{ }^{1}, \mathrm{HX} u^{1}$ and $\mathrm{L} \mathrm{Zhu}^{4}$
Although the pathogenesis of DED is not fully understood, more evidence indicates that this disease is potentially associated with immune and inflammatory processes, which consequently affect the ocular surface. ${ }^{2,3}$ Tear-deficient DE can be mainly categorized as Sjögren's syndrome DE (SSDE) and non-Sjögren's syndrome DE (NSSDE). All types of DED result in damage of the ocular surface epithelia and are consequently associated with ocular surface inflammation. ${ }^{4}$ Therefore, it is essential to understand the cause of DED so as to provide better therapeutic options.

The ongoing activation of pathogenic immune cells, primarily the CD4 ${ }^{+} \mathrm{T}$ cells, has a significant role in the pathogenesis of DED. 5,6 Th17 cells, the newly discovered subset of $\mathrm{CD}^{+}{ }^{+} \mathrm{T}$ cells, ${ }^{7,8}$ were recently found to participate in most ocular inflammatory diseases, such as uveitis, ${ }^{9,10}$ scleritis, ${ }^{10}$ and herpes virus-induced corneal inflammation. ${ }^{10}$ However, such effective T-cell response in DED has not been identified yet. Both IL-17 and IL-22 are the effective cytokines of Th17 cells, the levels of which, in tears, may represent the immune response of Th17 cells during the pathogenesis of DED. ${ }^{11-13}$

In this study, we evaluated the levels of IL-17 and IL-22 in tears of patients with Sjögren's or non-Sjögren's syndrome DED. In addition, the correlation between disease severity and IL-17 as well as IL-22 levels in patients was also analyzed.

Materials and methods

\section{Patient information}

This study was approved by local Ethics Committee of Wuxi No. 2, People's Hospital and the Nanjing Medical University. All donors provided consent before tear donation. In all, 60 
subjects were recruited from Wuxi No. 2, People's Hospital, between 2011 and 2012 including 20 healthy donors, 20 patients with NSSDE and 20 patients with SSDE. DE patients had typical signs and symptoms as defined by the International DE workshop report. ${ }^{14}$ Normal subjects without any ocular problems and systemic diseases voluntarily participated as controls. Exclusion criteria included any previous or present ocular disease other than DED, surgery, contact lens wearing, under systemic therapies within 3 months and topical therapies other than artificial tears for the previous 3 months.

\section{Clinical examination}

Clinical evaluations were performed following the sequence shown in Table 1, which included symptom questionnaire, tear film break-up time (TBUT) test, corneal fluorescein staining, and Schirmer I test.

Meibomian gland dysfunction (MGD) is also one of the leading causes of DE syndrome; however, the presence of MGD was not assessed in this study.

\section{Symptom questionnaire}

DE-related symptoms were evaluated with the help of the questionnaire. The questionnaire included eight most

Table 1 Clinical characteristics of the subjects enrolled in the study

\begin{tabular}{lccc}
\hline Parameters & Controls $(\mathrm{n}=20)$ & NSS $(\mathrm{n}=20)$ & SS $(\mathrm{n}=20)$ \\
\hline Age (years) & $54.2 \pm 7.3$ & $50.5 \pm 4.6$ & $52.5 \pm 8.1$ \\
Sex (M/F) & $14 / 6$ & $5 / 15$ & $9 / 11$ \\
Questionnaire score & $2.70 \pm 2.58$ & $26.55 \pm 11.67^{*}$ & $16.40 \pm 6.23^{* \#}$ \\
TBUT (s) & $15.15 \pm 2.05$ & $3.125 \pm 1.24^{*}$ & $2.90 \pm 1.50^{*}$ \\
Corneal staining (grade) & $0.25 \pm 0.23$ & $5.23 \pm 2.14^{*}$ & $5.93 \pm 2.76^{*}$ \\
Schirmer I test (mm) & $20.65 \pm 6.08$ & $4.60 \pm 1.75^{*}$ & $2.03 \pm 0.73^{* \#}$ \\
\hline
\end{tabular}

Abbreviations: F, female; $\mathrm{M}$, male.

All parameters are given as mean \pm SD. The statistical analysis ( $P$-value between study groups and controls is shown. ${ }^{*} P<0.05$, patients $v$ s controls; ${ }^{\#} P<0.05$, SS vs NSS.

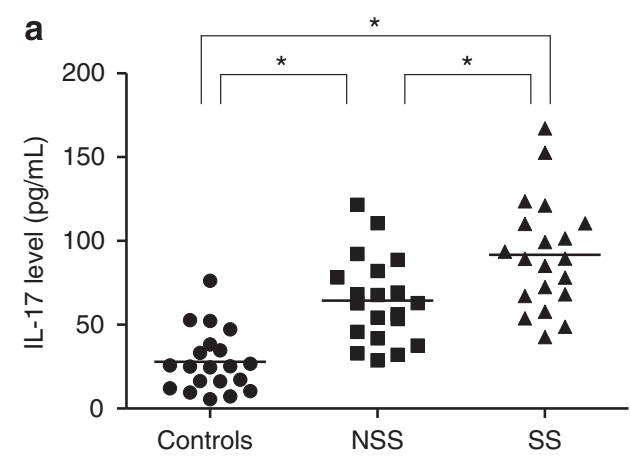

frequent symptoms of DE used in multi-center clinical trials of DED in China. Each symptom was scored from 0 to 9 , to yield a total score of 72 regarding eyestrain, dryness, sandy/gritty feeling, burning/stinging, sense of eye pressure, pain, sensitivity to light, and eye congestion.

\section{TBUT test}

Fluorescein strips (Jing Ming Inc., Tianjing, China) previously wetted with $0.9 \%$ sodium chloride were gently applied to the inferior fornix. Following instillation of fluorescein and a period of blinking, the TBUT was measured, particularly, the appearance of the first randomly distributed dry spot occurring in the interval between the last blink and the occurrence of break up.

\section{Corneal fluorescein staining}

Corneal integrity was evaluated with fluorescein strips (Jing Min, Inc.). The intensity of corneal fluorescein staining was recorded in each quadrant on the cornea (temporal, nasal, superior, and inferior) using a standardized 4 -point scale $(0=$ none, $1=$ mild, $2=$ moderate, and $3=$ severe). Total fluorescein scores were calculated as the sum of scores. ${ }^{15}$

\section{Schirmer I test}

The Schirmer I test was performed by placing one sterile strip (Schirmer Tear Test Strips, $5 \times 35$ mm; Jing Ming Inc.) in the lateral canthus of the inferior lid margin of both eyes without topical anesthesia. Participants were asked to close their eyes during the test and the length of wetting was measured in millimeters after 5 min. ${ }^{16}$

\section{Measurement of IL-17 and IL-22 levels in tears}

A previously reported protocol was followed for sampling tears. Minimally stimulated tear samples

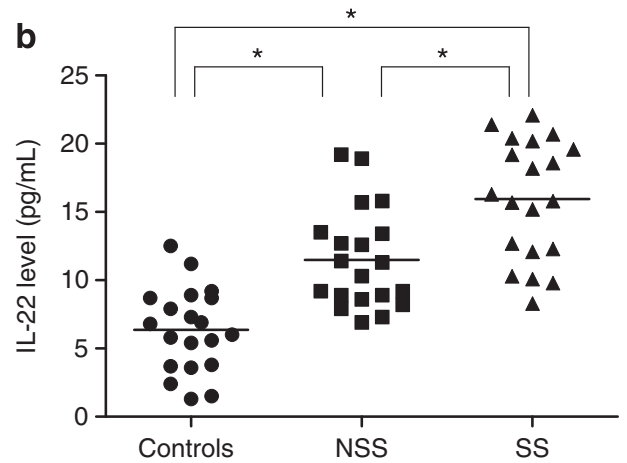

Figure 1 The levels of Th17-associated cytokines including IL-17 (a) and IL-22 (b) in the tears of controls and patients with or without Sjögren's syndrome. ${ }^{*} P<0.05$. 
were collected non-traumatically from the external canthus of open eyes between 0800 and 0900 hours by the same doctor avoiding additional tear reflex as much as possible. Glass capillary micropipettes $(5 \mu \mathrm{l})$ were used to collect $10 \mu \mathrm{l}$ tears from each eye of individual subject $(<1 \mu \mathrm{l} / \mathrm{min}$ ). Each sample was then transferred into a sterile microtube and stored at $-80^{\circ} \mathrm{C}$ until further examination. The concentrations of cytokines in the tears were measured using commercially available enzymelinked immunosorbent assay (ELISA) kit (R\&D Systems, Minneapolis, MN, USA) according to the manufacturer's instructions. The assay was performed in triplicates to ensure data reproducibility.

\section{Statistical analysis}

Data was shown as mean \pm SD. Unpaired Student's $t$-test and $\chi^{2}$-test were used to compare the ELISA results between the groups. Spearman correlation analysis was used to assess the relationship between cytokine levels and clinical parameters. A $P$-value of $<0.05$ was
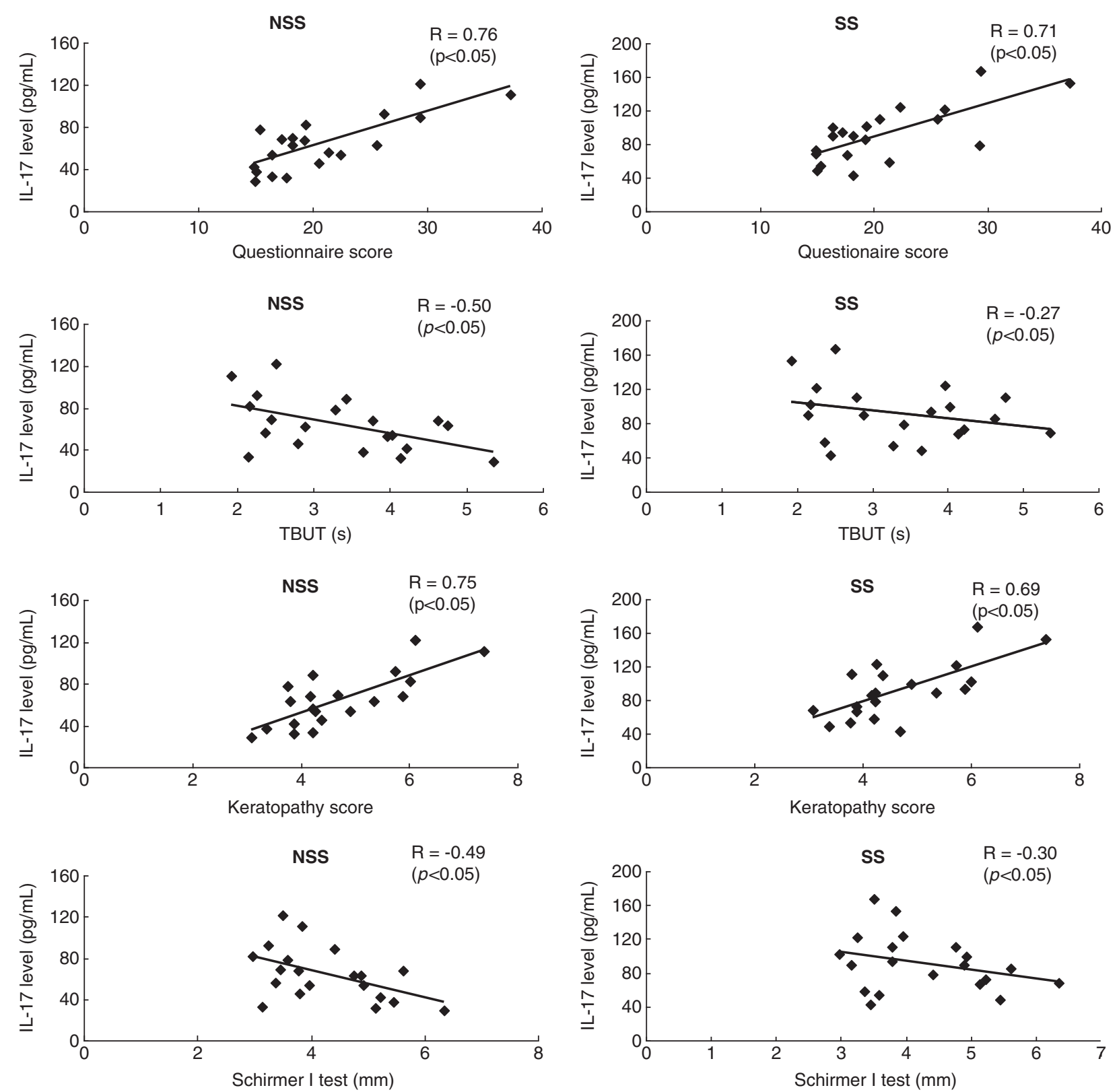

Figure 2 The correlation between IL-17 level in tears of DE patients with or without Sjögren's syndrome and clinical parameters including questionnaire score, TBUT, keratopathy score, and Schirmer I test. 
considered to be statistically significant. Analysis of data was performed using SPSS 15.0 (Chicago, IL, USA).

\section{Results}

\section{Characteristics of study population}

Patient information, questionnaire score and clinical parameters, including TBUT, corneal staining, and Schirmer I test with the controls, NSSDE and SSDE groups were shown in Table 1 . There were no significant differences observed in demographic features (including age and sex ratio) among the study groups $(P>0.05)$, but the questionnaire score and clinical parameters among DE patients were higher than those of controls $(P<0.05)$. In addition, between the NSSDE and SSDE patient group, the values of questionnaire score and Schirmer I test were significantly different.

\section{IL-17 and IL-22 levels in tears}

The tear levels of Th17-associated cytokines, particularly IL-17 and IL-22, in different groups are shown in Figure 1.
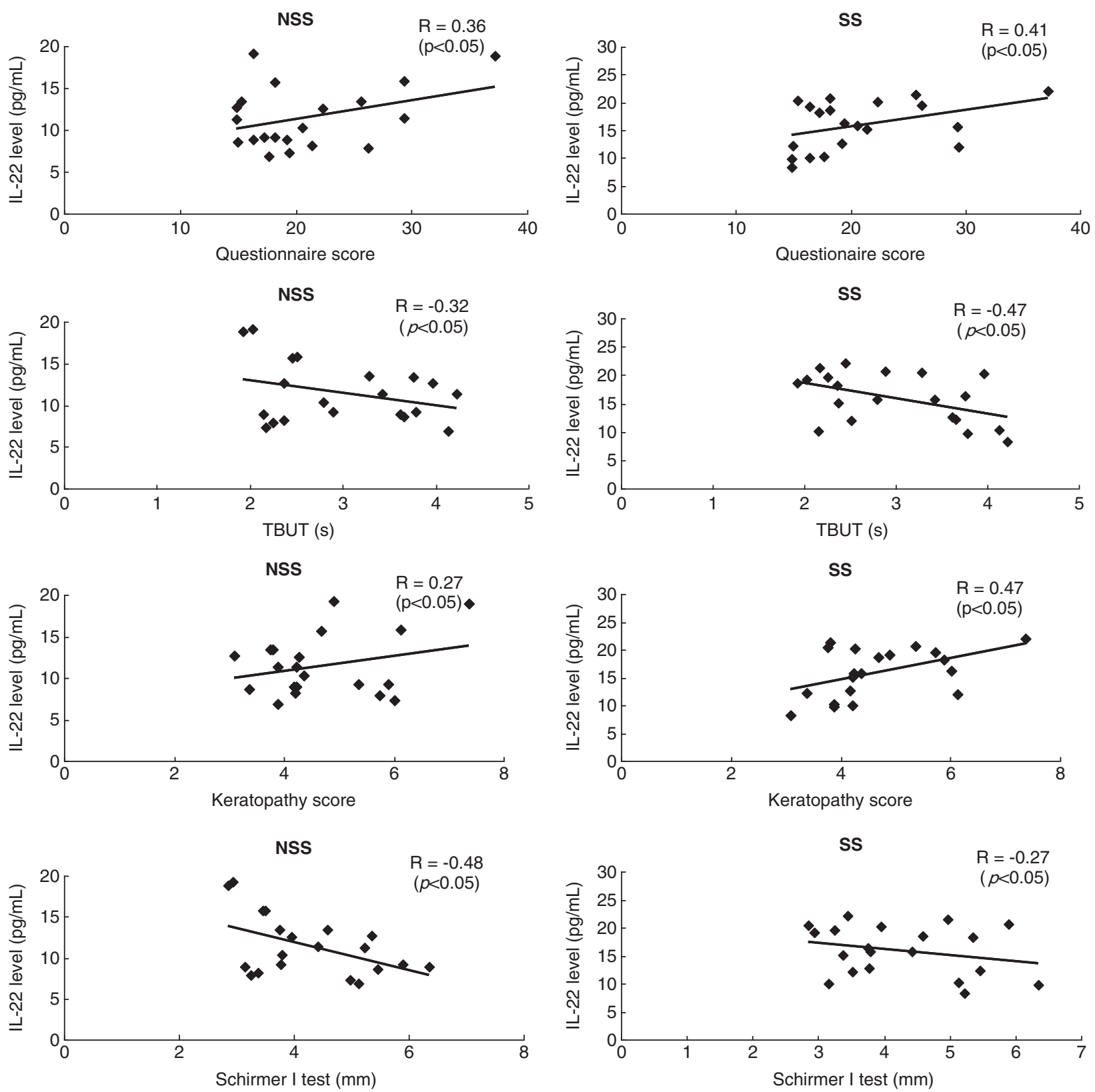

Figure 3 The correlation between IL-22 level in tears of DE patients with or without Sjögren's syndrome and clinical parameters including questionnaire score, TBUT, keratopathy score, and Schirmer I test. 
The levels of the two cytokines were significantly increased in the DE patient groups, when compared with those of the controls $(P<0.05)$. Furthermore, IL-17 and IL-22 levels in tears of SSDE patients were significantly higher than those of the NSSDE patients $(P<0.05)$.

\section{Correlation between cytokine levels and clinical parameters}

The correlation between IL-17 level in tears and clinical parameters in the DE patients with NSS or SS is presented in Figure 2. IL-17 level was positively correlated with questionnaire score and keratopathy score, but negatively correlated with TBUT and Schirmer I test in both NSSDE and SSDE patients $(P<0.05)$. The correlation between IL-22 level and clinical parameters was similar to the results of IL-17 $(P<0.05)$ (Figure 3$)$.

\section{Discussion}

In recent years, the incidence of DEs is increasing rapidly and this disease largely influences the quality of life in patients. Data indicated that about 33.7\% elderly Chinese people are suffering from the DED. ${ }^{17}$ Although the pathogenesis of DED is not well understood, abundant evidence from animal studies and clinical evaluations has suggested that inflammation significantly contributes to DED. ${ }^{18}$ Previous studies showed that inflammatory cytokines including IL-1, IL- 6 , IL- 8 , and TNF- $\alpha$, are important mediators in both human and animal DEDs. ${ }^{19-22}$ Recent reports indicated that pro-inflammatory cytokines were significantly increased in the conjunctiva tissue and tears of NSSDE and SSDE patients. ${ }^{21,22}$

Th17 cells are the newly recognized CD4 ${ }^{+} \mathrm{T}$-cell subset, which mainly produces cytokines, including IL-17A, IL-17F, and IL-22, and is involved in a wide range of autoimmune diseases. ${ }^{11-13}$ The level of IL-17 was found to be correlated with both activity and severity of various autoimmune diseases. Studies conducted on experimental mice model of DE have found that the level of IL-17 was significantly increased on the ocular surface and in tears. IL-22 has been shown to be pathogenically associated with several autoimmune diseases, including rheumatoid arthritis and non-autoimmune diseases such as respiratory-distress syndrome. However, little is known about the IL-17 and IL-22 levels in tears of DE patients.

In this study, we were the first to investigate the levels of IL-17 and IL-22 in tears of DE patients with or without Sjögren's syndrome. To avoid reflex tearing which might affect the results, minimally stimulated tear samples $(<1 \mu \mathrm{l} / \mathrm{min}$ ) were collected non-traumatically from the external canthus of open eyes between 0800 and 0900 hours by the same doctor. Furthermore, the correlation of cytokine levels with clinical parameters was also analyzed. Our results showed that the levels of IL-17 and IL-22 in tears were significantly increased in DE patients compared with those of controls and also higher in SSDE patients compared with those of NSSDE patients $(P<0.05)$. Increased levels of Th17-associated cytokines might have resulted from the stimulation of ocular surface epithelium during DED pathogenesis.

Moreover, IL-17 and IL-22 levels were positively correlated with questionnaire score and keratopathy score but negatively correlated with TBUT and Schirmer I test in both the NSSDE and SSDE patients $(P<0.05)$. These findings demonstrated that Th17 cells may have a key role in modulating the levels of IL-17 and IL-22 in DED pathogenesis.

In summary, our study found that ocular surface change in DED was associated with the increased levels of IL-17 and IL-22 in tears of DE patients. Additionally, IL-17 and IL-22 levels were correlated with clinical parameters measuring tear production and ocular surface integrity. These findings indicated that Th17 cells might be involved in the pathogenesis of DED. On the basis of these findings, our study significantly contributed to identification of novel therapeutic approaches in the treatment of DED.

\section{Summary}

What was known before

- DED is a complex, multifactorial disease which is accompanied by decreased tear secretion or increased tear evaporation. All forms of DE result in damage to the ocular surface epithelia and consequently associated with ocular surface inflammation. Therefore, it is essential to understand the causes of DED to provide better therapeutic options.

What this study adds

- Tears from DE patients contained significantly increased concentrations of IL-17 and IL-22 that showed correlation to severity of this disease. Therefore, Th17 cell-associated cytokines including IL-17 and IL-22 may have an important role in the immunopathogenesis of the DED.

\section{Conflict of interest}

The authors declare no conflict of interest.

\section{Acknowledgements}

We thank X Zhu for her assistance in the data analysis for comparing the ELISA results between the groups. The authors are very grateful to the Key Foundation of Nanjing Medical University (2010NJMUZ01), Guangzhou Manicipal Science and Technology Program (2010Y1-C081), National Natural Science Foundation (81300787) and the Natural Science Foundation of Jiangsu Province (BK2011168, BK2012105). 


\section{References}

1 The definition and classification of dry eye disease: report of the Definition and Classification Subcommittee of the International Dry Eye WorkShop (2007). Ocul Surf 2007; 5(2): 75-92.

2 Massingale ML, Li X, Vallabhajosyula M, Chen D, Wei Y, Asbell PA. Analysis of inflammatory cytokines in the tears of dry eye patients. Cornea 2009; 28(9): 1023-1027.

3 Yoon KC, Jeong IY, Park YG, Yang SY. Interleukin-6 and tumor necrosis factor- $\alpha$ levels in tears of patients with dry eye syndrome. Cornea 2007; 26(4): 431-437.

4 Pflugfelder SC. Anti-inflammatory therapy for dry eye. Am J Ophthalmol 2004; 137(2): 337-342.

5 Samarkos M, Moutsopoulos HM. Recent advances in the management of ocular complications of Sjögren's syndrome. Curr Allergy Asthma Rep 2005; 5(4): 327-332.

6 Sosroseno W, Herminajeng E. The role of T lymphocytes in Sjögren's syndrome. Asian Pac J Allergy Immunol 1997; 15(3): 167-176.

7 Harrington LE, Hatton RD, Mangan PR, Turner H, Murphy TL, Murphy KM et al. Interleukin 17-producing CD4 ${ }^{+}$effector $\mathrm{T}$ cells develop via a lineage distinct from the $\mathrm{T}$ helper type 1 and 2 lineages. Nat Immunol 2005; 6(11): 1123-1132.

8 Park $\mathrm{H}, \mathrm{Li} \mathrm{Z}$, Yang XO, Chang SH, Nurieva R, Wang $\mathrm{YH}$ et al. A distinct lineage of CD4 T cells regulates tissue inflammation by producing interleukin 17. Nat Immunol 2005; 6(11): 1133-1141.

9 Yoshimura T, Sonoda KH, Ohguro N, Ohsugi Y, Ishibashi T, Cua DJ et al. Involvement of Th17 cells and the effect of anti-IL-6 therapy in autoimmune uveitis. Rheumatology (Oxford) 2009; 48(4): 347-354.

10 Amadi-Obi A, Yu CR, Liu X, Mahdi RM, Clarke GL, Nussenblatt RB et al. TH17 cells contribute to uveitis and scleritis and are expanded by IL-2 and inhibited by IL-27/STAT1. Nat Med 2007; 13(6): 711-718.

11 Hashimoto T, Akiyama K, Kobayashi N, Mori A. Comparison of IL-17 production by helper T cells among atopic and non atopic asthmatics and control subjects. Int Arch Allergy Immunol 2005; 137(Suppl 1): 51-54.
12 Molesworth-Kenyon SJ, Yin R, Oakes JE, Lausch RN. IL-17 receptor signaling influences virus-induced corneal inflammation. J Leukoc Biol 2008; 83(2): 401-408.

13 Zheng Y, Danilenko DM, Valdez P, Kasman I, Eastham-Anderson J, Wu J et al. Interleukin-22, a T(H)17 cytokine, mediates IL-23-induced dermal inflammation and acanthosis. Nature 2007; 445(7128): 648-651.

14 Lemp MA. Report of the National Eye Institute/Industry workshop on clinical trials in dry eyes. CLAO J 1995; 21(4): 221-232.

15 Macri A, Rolando M, Pflugfelder S. A standardized visual scale forevaluation of tear fluoresecein clearance. Ophthalmology 2000; 107(7): 1338-1343.

16 Van Bijsterveld OP. Diagnostic tests in the Sicca syndrome. Arch Ophthalmol 1969; 82(1): 10-14.

17 Lin PY, Tsai SY, Cheng CY, Liu JH, Chou P, Hsu WM. Prevalence of dry eye among an elderly Chinese population in Taiwan: the Shihpai eye study. Ophthalmology 2003; 110(6): 1096-1101.

18 Research in dry eye: report of the research subcommittee of the International Dry Eye Workshop (2007). Ocul Surf 2007; 5(2): 179-194.

19 Solomon A, Dursun D, Liu Z, Xie Y, Macri A, Pflugfelder SC. Pro- and anti-inflammatory forms of interleukin-1 in the tear fluid and conjunctiva of patients with dry-eye disease. Invest Ophthalmol Vis Sci 2001; 42(10): 2283-2292.

20 Oshida T, Iwata M, Sakimoto T, Sawa M. Tumor necrosis factor-alpha in tears of patients with Sjogren's syndrome. Nihon Ganka Gakkai Zasshi 2004; 108(5): 297-301.

21 Luo L, Li DQ, Doshi A, Farley W, Corrales RM, Pflugfelder SC. Experimental dry eye stimulates production of inflammatory cytokines and MMP-9 and activates MAPK signaling pathways on the ocular surface. Invest Ophthalmol Vis Sci 2004; 45(12): 4293-4301.

22 Narayanan S, Corrales R, Farley W, McDermott AM, Pflugfelder SC. Interleukin-1 receptor-1-deficient mice show attenuated production of ocular surface inflammatory cytokines in experimental dry eye. Cornea 2008; 27(7): 811-817. 